\title{
NEIGHBORHOOD PRODUCTION STRUCTURES, WITH AN APPLICATION TO THE THEORY OF INTERNATIONAL TRADE*
}

\section{By RONALD W. JONES and HENRYK KIERZKOWSKI}

IT is a common observation that unless some structure is imposed, general equilibrium models of production with many factors and commodities yield few unambiguous comparative statics results. One example where structure allows such results is the higher-dimensional version of the specific-factors model, with the stark contrast between $n$ factors each tied to a separate production process and a single factor mobile among all activities supporting clear factor-price responses to changes in commodity prices. In the present paper we analyze a different kind of higher-dimensional structure, one in which no factor is specific to any single activity, but nonetheless occupational choices are severely limited. The structure is inspired by a model in which geographic proximity is crucial: each productive process uses as inputs only those factors located in its immediate "neighborhood".

The paper first develops some general properties of the $n$-factor, $n$-commodity model with neighborhood productive structures, with particular attention paid to the effects on factoral income distribution of changes in commodity prices. Two branches of the general model are distinguishedthe "cooperative" model, in which a single price rise in any sector jointly benefits the neighboring productive factors used as inputs to that sector, and the "non-cooperative" model, in which one factor gains and the other loses in the favored sector. The "non-cooperative" model is the one analyzed in this paper. A multilateral concept of factor intensity emerges as the crucial determinant of the winning factor in the favored sector. Further comparisons of factor intensity are developed viz., rankings within the two parts of the economy created when neighboring sectors share in a common price rise, the "favored" part comprising these neighboring sectors and the "fixed-price" part consisting of the rest of the economy.

Although the general model can be applied in several ways to various problems that are discussed in the theory of international trade, we choose to focus on one such problem that heretofore has received little attention. In a two-country model of the world economy suppose that trade is allowed not only in final commodities but also in two (both) types of sector-specific capital. This can be viewed as a particular example for a neighborhood production structure of the "non-cooperative" type with $n=4$. If world

\footnotetext{
* This research was initiated while Jones was a visitor at the Graduate Institute of International Studies in Geneva. He wishes to thank that Institute as well as the National Science Foundation for research support provided by Grant No. SES-8309386. Basic ideas for the paper were prompted by discussions of the earlier Cornes and Kierzkowski (1981) manuscript.
} 
taste patterns change in favor of the type of commodity produced in each country using a particular type of internationally mobile capital, should we expect the return to that capital unambiguously to rise, the return to the other kind of capital to fall, with less severe adjustments required in each country's wage rate? As we demonstrate, such a result is by no means assured. Furthermore, much depends upon intra-industry factor intensity comparisons between countries rather than traditional inter-industry rankings between commodities within either country. Indeed, the neighborhood production structure reveals how such a taste change might unambiguously favor real wages at the expense of both types of capital even when the commodity that has gone up in price in each country is capital-intensive.

The final section of the paper offers some concluding remarks about the relationship between the model exhibiting the neighborhood production structure and the sector-specific model.

\section{Neighborhood production structures: general properties}

In the $n$-sector version of the model there exist $\underline{n}$ distinct productive activities, each only employing two different factors of production. Symmetry is further reflected in the requirement that every productive factor has two alternative employment outlets. The schematic illustration in Fig. 1 shows each producing sector, $x_{j}$, geographically located on a circle, with each sector making use of the two neighboring factors shown by the arcs connecting the $x_{j}$ 's. Thus $x_{2}$ employs factors $V_{2}$ and $V_{3}$ while factor $V_{1}$ has two sectors in which it finds work: $x_{n}$ and $x_{1}$. No productive factor is completely specific, but mobility is restricted for each factor to the two neighboring productive sectors. Whereas in the general $n \times \underline{n}$ model of production each factor is potentially employable in all $\underline{n}$ sectors, in the

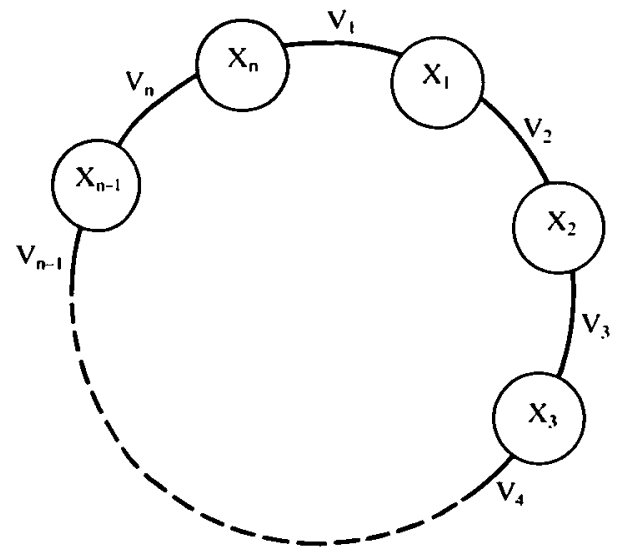

FIG. 1 
neighborhood production structure this mobility is limited to the two nearby sectors for each and every factor. Thus the neighborhood structure adopts the idea that each factor may not be required in all activities and pushes this idea to the limit that still allows some element of choice for all factors and all commodities.

Suppose the price of commodity $j$ rises. In a competitive market the return to at least one of the factors employed in $x_{j}$ must rise, and perhaps both do. The model featuring a neighborhood production structure allows each of these outcomes. The "cooperative" result, wherein a price rise rebounds to the benefit of both factors employed in the favoured industry, is a feature of models in which the number of producing sectors, $\underline{n}$, is odd, whereas "non-cooperative" outcomes characterize models in which $n$ is even. (The $2 \times 2$ Heckscher-Ohlin model is the simplest case-the StolperSamuelson feature of a price rise leading to a real gain for one factor and loss for the other is standard for that case.) These results follow immediately from a phenomenon characteristic of both models of this type: the ripple effect for the factor returns in that part of the economy in which commodity prices have not changed.

Let the industry favored by the price rise be the last one, sector $\underline{n}$. If the return to the first factor, $w_{1}$, rises, the return to other factor employed in the constant-price first sector, $w_{2}$, must fall, leading to a balancing rise in $w_{3}$ since $p_{2}$ is assumed constant, and a fall in $w_{4}$, etc. In any competitive model in which some commodity prices are constant, those factors employed in the constant-price sectors which experience an increase in returns must, by the competitive profit conditions, force other factor returns to fall. In the neighborhood production structure these balancing forces result in ripples of factor-price increases and decreases for alternatively sequenced factors. Thus, in the case in which only $p_{n}$ rises, if $w_{1}$ rises and $n$ is odd, so does $w_{n}$. Factors $V_{1}$ and $V_{n}$, the pair used in the favored $n$th sector, share cooperatively in the rise in $p_{n}$. But, should $n$ be an even number, an increase in $w_{1}$ sends ripples throughout the rest of the economy consistent only with a fall in $w_{n}$. The increase in $p_{n}$ cannot be shared cooperatively by the two factors employed in producing $x_{n}$. Of course this argument only states that $w_{1}$ and $w_{n}$, the returns to the two factors employed by favored-sector $\underline{n}$, must move in opposite directions. Which of the two factors gains depends upon factor intensities.

A principal result to be established now is that if the number of productive sectors, $\underline{n}$, is even, an increase in $p_{j}$ (alone) will serve to increase the return to factor $j$, (and reduce the return to factor $j+1$, the other factor employed in the $j$ th sector) if and only if commodity $j$ is relatively intensive in its use of factor $j$. But a multilateral concept of factor intensity is required, one that contains information on all sectors of the economy. To proceed formally, let $\hat{w}$ represent the vector of relative changes in factor prices, $\hat{p}$ the vector of relative changes in commodity prices, and $\theta_{i j}$ the distributive share of the earnings of factor $\underline{i}$ in sector $\underline{j}$. By the competitive 
profit conditions,

$$
\left[\begin{array}{ccccc}
\theta_{11} & \theta_{21} & 0 & \ldots & 0 \\
0 & \theta_{22} & \theta_{32} & \ldots & 0 \\
0 & 0 & \theta_{33} & \ldots & 0 \\
& & & & \\
& & & \theta_{n-1, n-1} & \theta_{n, n-1} \\
\theta_{1 n} & 0 & 0 & 0 & \theta_{n n}
\end{array}\right] \hat{w}=\hat{p}
$$

The determinant of coefficients, $|\theta|$, is shown in (2):

$$
|\theta|=\prod_{j} \theta_{j j}-\prod_{j} \theta_{j+1, j} \text { where } j+1=1 \text { for } j=n .
$$

Suppose only $p_{n}$ rises. Then the solution for $\hat{w}_{n}$ is shown in (3):

$$
\hat{w}_{n}=\frac{1}{|\theta|} \prod_{j \neq n} \theta_{j j} \cdot \hat{p}_{n}
$$

Therefore the sign of the determinant, $|\theta|$, dictates the fate of factor $\underline{n}$ when the price of commodity $\underline{n}$ rises when $\underline{n}$ is even. ${ }^{1}$

The multilateral concept of factor intensity appropriate for models with neighborhood productive structures is that sector $j$ uses factor $j$ intensively in a multilateral sense if and only if $|\theta|$ is positive. (Note what this entails: If sector $j$ employs factor $\underline{j}$ intensively in this multilateral sense, then sector $\underline{i}$ employs factor $\underline{i}$ intensively, for all $\underline{i}$. No two sectors can have a multilateral intensity in the same factor.) Consider the $n$th sector, which uses factors $V_{n}$ and $V_{1} . V_{n}$ is also used to produce $x_{n-1}$ and $V_{1}$ to produce $x_{1}$. The economy can be considered to be aggregated into two parts: favored-sector $n$ and the rest of the economy, and the latter also employs $V_{n}$ and $V_{1}$ (and all other $V_{j}$ as well). $|\theta|$ is positive if and only if

$$
\frac{\theta_{n n}}{\theta_{1 n}}>\frac{\theta_{n, n-1}}{\theta_{n-1, n-1}} \cdot \frac{\theta_{n-1, n-2}}{\theta_{n-2, n-2}} \ldots \frac{\theta_{21}}{\theta_{11}}
$$

Each $\theta_{i j}$ equals $w_{i} a_{i j} / p_{j}$, so that the inequality in (4) can be rewritten as in $\left(4^{\prime}\right)$ :

$$
\frac{a_{n n}}{a_{1 n}}>\frac{a_{n, n-1}}{a_{n-1, n-1}} \cdot \frac{a_{n-1, n-2}}{a_{n-2, n-2}} \ldots \frac{a_{21}}{a_{11}}
$$

Both sides of this inequality have dimensionality factor $n$ per unit of factor 1 and thus involve a comparison of sector $\underline{n}$ 's use of $V_{n}$ and $V_{1}$ relative to the rest of the economy's use of these two factors. The logic and interpretation of this argument are analogous to the problem of assigning commodities to

${ }^{1}$ If $\underline{n}$ is odd, the expression for $|\theta|$ becomes $\left(\prod_{j} \theta_{j j}+\prod_{j} \theta_{j+1, j}\right)$ which must be positive. In this paper we restrict our attention to "non-cooperative" models in which $\underline{n}$ is even. 
countries according to a multilateral criterion for comparative advantage in a Ricardo-Graham model of trade. ${ }^{2}$

To pursue the idea of separating the economy into two parts (favored sector $\underline{n}$ where price has risen and sectors 1 through $\underline{n}-\underline{1}$ where prices are constant), consider the competitive profit conditions of change in the constant-price sectors. These are shown by the first $\underline{n}-1$ equations in (1). The first of these can be solved for $\hat{w}_{1}$ in terms of $\hat{w}_{2}$ :

$$
\hat{w}_{1}=-\frac{\theta_{21}}{\theta_{11}} \hat{w}_{2}
$$

The second can, in similar fashion, be solved for $\hat{w}_{2}$ in terms of $\hat{w}_{3}$ and, continuing this way, the $(n-1)$ st equation shows that:

$$
\hat{w}_{n-1}=-\frac{\theta_{n, n-1}}{\theta_{n-1, n-1}} \hat{w}_{n} .
$$

Successive substitutions allow us to solve for $\hat{w}_{1}$ in terms of $\hat{w}_{n}$ :

$$
\hat{w}_{1}=(-1)^{n-1} \frac{\prod_{j \neq n} \theta_{j+1, j}}{\prod_{j \neq n} \theta_{j j}} \hat{w}_{n} .
$$

Now rewrite this expression together with that for sector $\underline{n}$ (which has risen in price) as equation set (5):

$$
\begin{gathered}
\left(\prod_{j \neq n} \theta_{j j}\right) \hat{w}_{1}+(-1)^{n}\left(\prod_{j \neq n} \theta_{j+1, j}\right) \hat{w}_{n}=0 \\
\theta_{1 n} \hat{w}_{1}+\theta_{n n} \hat{w}_{n}=\hat{p}_{n}
\end{gathered}
$$

This aggregation of sectors 1 through $(n-1)$ allows us to represent it as a part of the economy using factors $V_{1}$ and $V_{n}$ just as does section $n$. In non-cooperative models in which $n$ is even, the typical two-by-two result is obtained wherein an increase in $p_{n}$ raises, by a magnified amount, the return to the factor used intensively in that sector and lowers the return to the other factor. The concept of factor intensity relevant here, of course, is the sign of $|\theta|$ in (2), which is the determinant of coefficient in (5).

\section{Neighboring price rises in non-cooperative models}

For some purposes it is useful to investigate the impact on the factoral distribution of income when two neighboring sectors both share in a rise in price (of the same relative magnitude) while prices remain constant in the rest of the economy. For example, let sectors $n$ and 1 share a common price rise: $\hat{p}_{n}=\hat{p}_{1}=\hat{p}$. Factors in the economy can then be grouped into three categories: (a) A single factor, $V_{1}$, is "specific" to the two sectors which have experienced a price rise; (b) Two "edge" factors, $V_{n}$ and $V_{2}$, are used both in that part of the economy favored by the price rise $\left(x_{n}\right.$ and $\left.x_{1}\right)$ and in

${ }^{2}$ See the discussion in Jones (1961). 
the rest of the economy; and (c) The remaining factors $V_{3}, \ldots, V_{n-1}$, are used exclusively in sectors which have not experienced a price rise. For these factors, the ripple effect described earlier can be expected to hold: if $w_{2}$ rises, $w_{3}$ will fall, $w_{4}$ will rise, and so on, with $w_{n-1}$ falling (since $\underline{n}$ is an even number by assumption).

With the qualitative similarity in the experience of the two "edge" factors established, it becomes tempting to ask the circumstances under which factor price responses to neighboring price rises in non-cooperative models resemble those of the specific-factor, $(n+1) \times n$ model. In particular, with $p_{n}$ and $p_{1}$ rising, can (a) the return to factor 1 , that factor used exclusively (or "specifically") in the favored sectors of the economy, rise relatively more than $p_{n}$ and $p_{1}$, (b) the return to "edge" factors $\underline{n}$ and 2 behave like "mobile" factors (these are used partly in the favored sectors and partly in the rest of the economy) and rise, but by less than $p_{n}$ and $p_{1}$, with (c) some aggregate measure of the returns to all other factors falling? (These other factors are "specific" to that part of the economy not experiencing a price rise; of course the ripple effect precludes all these factor prices from falling).

Equation set (1) can be solved explicitly for the factor price changes associated with a rise in the price of neighboring sectors $\underline{1}$ and $\underline{n}$. But before proceeding in this formal fashion consider the potential role of factor intensities. The multilateral ranking for all $\underline{n}$ sectors taken together required a comparison between $\Pi \theta_{j j}$ and $\Pi \theta_{j+1, j}$. The former was assumed to be larger $(|\theta|>0$ in equation (2)). Neighboring price rises for sectors 1 and $n$ serve to split the economy into two parts: the "favored" part (sectors 1 and $n$ ), and the "fixed price" part (sections $j \neq 1, n$ ). Suppose a similar comparison of the product of distributive shares were to be made within each such part of the economy. That is, suppose $\prod_{j=1, n} \theta_{j j}$ were compared with $\prod_{j=1, n} \theta_{j+1, j}$ for the favored part of the economy, while $\prod_{j \neq 1, n} \theta_{i j}$ were compared with $\prod_{j \neq 1, n} \theta_{j+1, j}$ for the fixed-price part. As we now show, such separate comparisons prove crucial in determining the effect of these neighboring commodity price changes on factor prices.

Consider, first, the significance of the intensity ranking within the favored part of the economy consisting of sectors $\underline{1}$ and $\underline{n}$. A comparison of $\prod_{j=1, n} \theta_{j j}$ with $\prod_{j=1, n} \theta_{j+1, j}$ boils down to a comparison of the intensity with which factor 1 is used in sectors 1 and $n$ ( $\theta_{11}$ compared with $\left.\theta_{1 n}\right)$. What is the same thing, this involves a comparison of the intensity with which "edge" factor $\underline{n}$ is used in that sector of the favored part of the economy in which it is employed $\left(\theta_{n n}\right)$, with the intensity with which the other "edge" factor, 2 , is used in that sector of the favored part of the economy in which it is employed $\left(\theta_{21}\right)$. With $\hat{p}_{1}$ and $\hat{p}_{n}$ both set equal to a common (positive) $\hat{p}$, equations (6) display the competitive profit conditions of change in the two 
favored sectors:

$$
\begin{aligned}
& \theta_{11} \hat{w}_{1}+\theta_{21} \hat{w}_{2}=\hat{p} \\
& \theta_{1 n} \hat{w}_{1}+\theta_{n n} \hat{w}_{n}=\hat{p}
\end{aligned}
$$

Without further knowledge of the structure of the rest of the economy it is impossible to tell from the conditions in the favored part of the economy alone whether $\hat{w}_{1}$ exceeds $\hat{p}$ (with "edge" $\hat{w}_{2}$ and $\hat{w}_{n}$ falling short of $\hat{p}$ ) or whether positions are reversed so that both $\hat{w}_{2}$ and $\hat{w}_{n}$ exceed $\hat{p}$ (with $\hat{w}_{1}$ less than $\hat{p}$ ). Nonetheless, the factor-intensity ranking within the favored part of the economy does indicate which of $\hat{w}_{2}$ and $\hat{w}_{n}$ lies closer to $\hat{p}$. Thus if $\theta_{n n}$ exceeds $\theta_{21}, \hat{w}_{n}$ must lie closer to $\hat{p}$ than does $\hat{w}_{2}$ because $\hat{w}_{1}$ has a smaller share in sector $\underline{n}$ than it does in sector 1 . As a consequence, the comparison of $\prod_{j=1, n} \theta_{j j}$ with $\prod_{j=1, n} \theta_{j+1, j}$ in the favored part of the economy indicates which of the "edge" factors lies closer to the price change common to all (both) sectors in the favored part. In particular, the assumption that $\prod_{j=1, n} \theta_{i j}$ exceeds $\prod_{j=1, n} \theta_{j+1, j}$ implies either that $\hat{w}_{2}>\hat{w}_{n}>\hat{p}>\hat{w}_{1}$ or that $\hat{w}_{1}>\hat{p}>\hat{w}_{n}>\hat{w}_{2}$.

Somewhat the same procedure can be followed in the fixed-price part of the economy, with one exception. With $\underline{n}$ large, more than two sectors comprise this subset of the economy, so that a multilateral ranking is required, involving a comparison between $\Pi \theta_{i j}$ over all industries in the fixed-price part $(j \neq 1, n)$, on the one hand, and $\prod_{j \neq 1, n} \theta_{j+1, j}$, on the other. To see this, follow the procedure used in obtaining equation (5), which revealed the ripple effect connecting the returns to the two edge factors. Substitution through the competitive profit conditions for sectors 2 through $n-1$ reveals that

$$
\hat{w}_{2}=(-1)^{n-2} \frac{\prod_{j \neq 1, n} \theta_{j+1, j}}{\prod_{j \neq 1, n} \theta_{j j}} \cdot \hat{w}_{n} .
$$

In non-cooperative models with an even number of sectors (or, more particularly, an even number of sectors in the fixed-price part of the economy), edge returns rise or fall together. What is crucial is which change in factor return lies closer to zero (the value of the commodity price change in the fixed-price part of the economy). By analogy with our remarks about the favored part of the economy we would like to define edge factor 2 as used intensively in a multilateral sense in the fixed-price part of the economy (which includes sector 2 ) as compared with edge factor $\underline{n}$ (where sector $\underline{n}$ is not included in the fixed-price part of the economy) if $\prod_{j \neq 1, n} \theta_{j j}$ exceeds $\prod_{j \neq 1, n} \theta_{j+1, j}$. As (7) reveals, if in this sense the fixed-price part of the economy uses factor 2 intensively, $\hat{w}_{2}$ lies closer to the commodity price change (zero) than does $\hat{w}_{n}$. 
Three factor intensity rankings have been considered: (i) The overall multilateral ranking in which we assume factors are numbered so that $\Pi \theta_{i j}$ exceeds $\Pi \theta_{j+1, j}$ and thus factor $\underline{j}$ is used intensively in industry $\underline{j}$; (ii) The bilateral comparison in the part of the economy consisting of neighboring sectors 1 and $n$ when they are the only sectors favored by a (similar) price rise; and (iii) The multilateral criterion in the fixed-price part of the economy, involving a comparison of reduced products $\prod_{j \neq 1, n} \theta_{i j}$ and $\prod_{j \neq 1, n} \theta_{j+1, j}$. We now define these latter two rankings as "consistent" with the assumption that overall $\Pi \theta_{j j}$ exceeds $\Pi \theta_{j+1, j}$ if, for the favored part, $\prod_{j=1, n} \theta_{j j}$ exceeds $\prod_{j=1, n} \theta_{j+1, j}$ and, for the fixed-price part, $\prod_{j \neq 1, n} \theta_{j j}$ exceeds $\prod_{j \neq 1, n} \theta_{j+1, j}$ More generally, the multilateral intensity ranking uniquely associates each factor with one of its neighboring sectors. When the economy is subdivided into two parts "consistency" follows if each edge factor is intensive in the subdivision containing the sector with which it has the multilateral association.

That these intensity comparisons matter is reflected in the formal solutions (from (1)) for the change in the returns to edge-factors 2 and $n$ when $\hat{p}_{1}=\hat{p}_{2}=\hat{p}$ :

$$
\begin{gathered}
\frac{\hat{w}_{2}}{\hat{p}}=\frac{\left(\theta_{n n}-\theta_{21}\right)}{|\theta|} \prod_{j \neq 1, n} \theta_{j+1, j} \\
\frac{\hat{w}_{n}}{\hat{p}}=\frac{\left(\theta_{n n}-\theta_{21}\right)}{|\theta|} \prod_{j \neq 1, n} \theta_{j j}
\end{gathered}
$$

As well, we can exhibit the change in the real return to the first factor, which is used only in the favored part of the economy:

$$
\frac{\hat{w}_{1}-\hat{p}}{\hat{p}}=\frac{\theta_{21} \theta_{n n}}{|\theta|}\left\{\prod_{j \neq 1, n} \theta_{j i}-\prod_{j \neq 1, n} \theta_{j+1, j}\right\}
$$

From these expressions it is clear that if intensity rankings in both subdivisions of the economy are consistent with the overall ranking, the factor price response to a neighboring commodity price rise in sectors 1 and $n$ is similar to that found in specific-factor models: ${ }^{3}$

$$
\hat{w}_{1}>\hat{p}>\hat{w}_{n}>\hat{w}_{2}>0>\sum_{i=3}^{n=1} \theta^{i} \hat{w}_{i} .
$$

\footnotetext{
${ }^{3}$ In the last term, $\theta^{i}$ refers to the share of factor $i$ in the national income. That this weighted sum for changes in prices of factors used exclusively in the fixed-price part of the economy must be negative can be simply proved by considering the competitive profit conditions of change in sectors 2 through $n-1$ (see equation (1)). Multiply each by the industry share of output $j$ in the national income, $\theta_{j}$, and add to obtain: $\left\{\theta_{2} \theta_{22} \hat{w}_{2}+\theta_{n-1} \theta_{n, n-1} \hat{w}_{n}\right\}+$ $\left\{\sum_{i=3}^{n-1} \theta^{i} \hat{w}_{i}\right\}=0$. Since with consistent rankings $\hat{w}_{2}$ and $\hat{w}_{n}$ are positive, the last inequality in (11) must follow.
} 
The logic behind this ranking can be revealed by noting that the only way $\hat{w}_{n}$ can lie closer than $\hat{w}_{2}$ to $\hat{p}$, (which it must do if $n$ is used bilaterally more intensively in the favored part than is 2) and, simultaneously, $\hat{w}_{2}$ lie closer than $\hat{w}_{n}$ to zero (which it must do if 2 is used more intensively in a multilateral sense in the fixed-price part than is $\underline{n}$ ) is for both to lie trapped between the two commodity price changes. The other factor price changes follow accordingly, since each commodity price change is flanked by factor price changes.

"Consistency" in the three factor-intensity rankings is not a required feature of the neighborhood production structure. To see what violation of consistency entails, we turn now to a particular application of the non-cooperative neighborhood model for $n=4$.

\section{International factor mobility and the case of $n=4$.}

The specific-factors model has been used extensively in the theory of international trade, especially in a small country setting in which commodity prices in two sectors are determined in the rest of the world, the nation's labor force is mobile between the two sectors, and two types of capital are specific in their use. Figure 2 provides a schematic illustration of a two-country world in which products from two industries are freely traded. Commodities $X$ and $\underline{X}^{*}$ are not identical, but they belong to the same $X$-industry, characterized in particular by the use in production of the same type of capital. Similarly, although the home country's $Y$-product may be differentiated by consumers from the foreign country's $\underline{Y}^{*}$, these two products are each produced by combining labor with a specific $Y$-type of
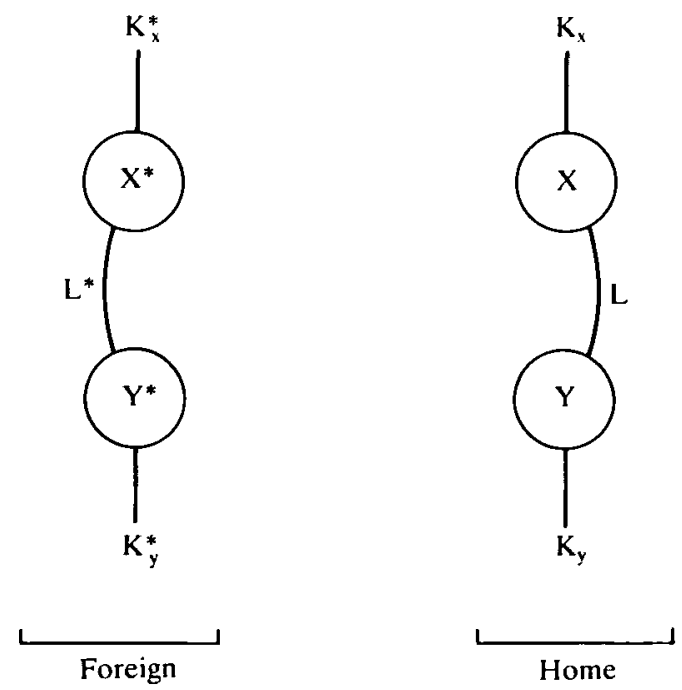

FIG. 2 


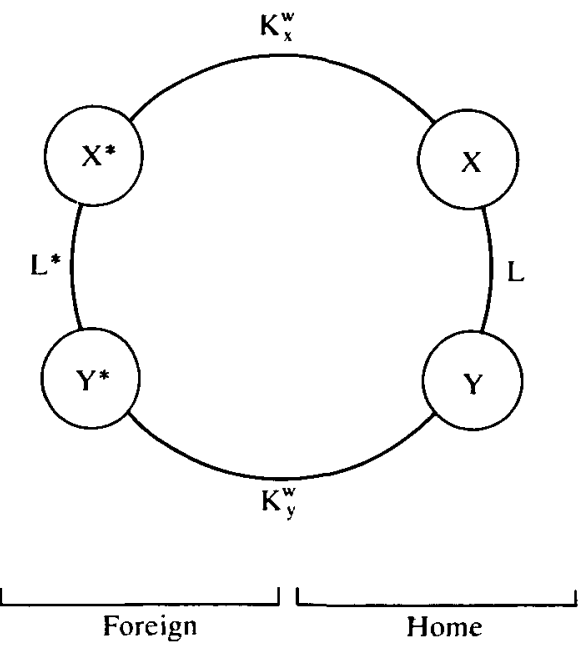

FIG. 3

capital. If, as in Fig. 2, no factor is internationally mobile, a shift in world taste patterns away from products of the world's $Y$-industry towards $X$-type products has a readily-identified impact on factor prices: in each country the return to type- $X$ captial unambiguously rises, that of type- $Y$ capital falls, while wage rates in each country rise relatively less than the price of $X$ but improve in terms of $\underline{Y}$.

The possible international mobility of capital alters this scenario. Of particular interest here is the case in which both types of sector-specific capital are internationally mobile. ${ }^{4}$ The schematic representation in Fig. 3 suggests that a trading world in which national labor forces are mobile within countries but immobile between, whereas capital is specific in its occupational use but footloose in world markets, provides a special example of a neighborhood production structure for $n=4$. Among the four producing sectors two types of commodities are distinguished: a world $X$-sector comprises products $X$ and $X^{*}$ while each country produces as well a product in the world $Y$-industry. World demands are such as to support an

\footnotetext{
${ }^{4}$ Some analyses, e.g., Brecher and Findlay (1983) and Jones and Dei (1983), consider the case in which one type of capital is internationally mobile. The effect in such a model of a rise in the world's relative price of commodity $\underline{X}$ is similar to that just described when there is no international factor mobility. Both types of capital are internationally mobile in Caves (1971) and Jones, Neary, and Ruane (1983), models concerned with the phenomenon of cross-hauling of capital. In the latter paper one of the commodities is treated as non-tradeable so as to avoid the possible necessity of specialization in one traded good for a small country which accepts commodity prices and the rate of return to internationally-mobile capital as given from the rest of the world. In our present treatment this is not necessary since rates of return are endogeneously determined and consumer tastes allow product differentiation within each industry, although both countries use the same industry-specific capital input.
} 
initial equilibrium in which all four products are produced. ${ }^{5}$ As before, suppose tastes shift towards products of the world's $X$-industry. In particular, consider an equiproportionate rise in world prices $p_{x}$ and $p_{x}^{*}$ (equal to $\hat{p}_{x}$ ), at the expense of constant prices throughout the world's $Y$-industry.

The simplification which the reduction of $\underline{n}$ to 4 introduces into our earlier analysis is that the "favored" and "fixed-price" parts of the economy each symmetrically comprise only two sectors. When the world prices of commodities $X$ and $\underline{X}^{*}$ rise, the "favored" part of the world economy consists of sector $X$ at home and sector $X^{*}$ abroad. The factor "specific" to this part is $K_{X}^{W}$, the world's supply of capital used only in the world's $X$-industry. The two "edge" factors are the labor forces in each country, $L$ and $L^{*}$. In the fixed-price part of the world economy, consisting of sectors $\underline{Y}$ at home and $\underline{Y}^{*}$ abroad, the "specific" factor is $Y$-type capital, $K_{Y}^{W}$. In the general case with many sectors $(j=2, \ldots, n-1)$ comprising the fixed-price part of the economy, a multilateral factor intensity ranking between edge factors involves the comparison of $\prod_{j \neq 1, n} \theta_{j j}$ with $\prod_{j \neq 1, n} \theta_{j+1, n}$ (see equation (7)). In our present case this reduces to a comparison between $\theta_{L Y} \theta_{K Y}^{*}$ and $\theta_{L Y}^{*} \theta_{K Y}$, a bilateral comparison of capital and labor shares in home and foreign $Y$-industries. ${ }^{6}$ Similarly, in the favored $X$-part of the world economy, the crucial bilateral intensity comparison is intra-industry (and inter-national), that between $\theta_{K X}$ and $\theta_{K X}^{*}{ }^{7}$

Our preceding remarks concerning Fig. 2's illustration of trade between two $3 \times 2$ economies with internationally immobile capitals have suggested that when world price rises in the $X$-industry, it might be reasonable to suppose that the return to type- $X$ capital rises by more than the price of $X$, the return to type- $Y$ capital falls, while both national wage rate changes lie trapped between the two price changes. Indeed, our general discussion of

\footnotetext{
${ }^{5}$ The world transformation surface in the space of the four commodities is strictly bowed out if, as we assume, techniques required to produce goods are different $(|\theta|$ different from zero). Therefore there is some set of relative commodity prices that will support positive production of all four commodities. If this set includes values for which $p_{x}=p_{x}^{*}$ and $p_{y}=p_{y}^{*}$, consumers might view products $X$ and $X^{*}$ in the two countries as identical (and similarly for $Y$ and $Y^{*}$ ). We adopt a more general stance: $X$ and $X^{*}$ can be members of the same industry, both requiring the same $K_{X}^{W}$, but differentiated in the eyes of the world's consumers, so that indifference surfaces may be strictly bowed in. Our assumption in any case is that initial equilibrium is at a price vector supporting positive production of all four commodities.

${ }^{6}$ In the present case translation requires factor 1 to be type- $X$ capital, factor 2 to be home labor, and factor $n$ to be foreign labor. Similarly, commodity 1 is the home $X$-sector and commodity $n$ the foreign $X^{*}$-sector. Compare Figs. 1 and 3.

${ }^{7}$ Comparing value shares for the same industry between countries (say $\theta_{K X}$ and $\theta_{K X}^{*}$ ) is not the same as comparing physical capital/labor ratios, since wage rates are presumably different. For example, if the home country has a lower level of techniques. expressing itself in a lower wage rate, the home country may have its $X$-sector capital-intensive compared with $X^{*}$ (in the sense of $\theta_{K X}$ greater than $\theta_{K X}^{*}$ ) but nonetheless employ a lower capital/labor ratio. Unless otherwise indicated, we always refer to factor intensities in the value sense imparted by distributive shares.
} 
neighboring price rises in a model with even $n$ has provided the conditions sufficient to guarantee this result in the present case in which both types of capital are internationally mobile: the intensity rankings in each part of the world economy be consistent with the overall multilateral factor intensity ranking.

In the general case we arbitrarily assumed that in a multilateral sense each industry used intensively that factor with the same number, so that $\Pi \theta_{j j}$ exceeds $\Pi \theta_{j+1, j}$. The comparison of Figs. 1 and 3 shows that such an assumption in our present interpretation with $n=4$ implies that $^{8}$

$$
\theta_{K X} \theta_{L Y} \theta_{K Y}^{*} \theta_{L X}^{*}>\theta_{L X} \theta_{K Y} \theta_{L Y}^{*} \theta_{K X}^{*}
$$

This implies that in the neighborhood production structure there must exist the factor-intensity reversal phenomenon for multilateral rankings. If the home $X$-sector is (multilaterally) capital intensive, the foreign $X^{*}$-sector is (multilaterally) labor intensive. Both $\underline{X}$ and $X^{*}$ share a common pool of capital, and they cannot both be intensive (multilaterally) in its use. Similarly, equation (12) states that the $Y$-sector at home is (multilaterally) labor intensive, but abroad $\underline{Y}^{*}$ is capital intensive.

"Consistency" for the experiment in which the prices of both $X$-sectors alone rise in unison requires that if (12) reflects the multilateral ranking, it is also the case that

$$
\theta_{K X} \theta_{L X}^{*}>\theta_{L X} \theta_{K X}^{*} \text { and } \theta_{L Y} \theta_{K Y}^{*}>\theta_{K Y} \theta_{L Y}^{*}
$$

or, more briefly, that $\theta_{K X}$ exceeds $\theta_{K X}^{*}$ and $\theta_{K Y}^{*}$ exceeds $\theta_{K Y}$. Indeed, one could start with the separate bilateral rankings: consistency requires that neither country have both its sectors more capital-intensive than the other country in a bilateral sense. If the $X$-industry at home is (in a bilateral value sense) more capital intensive than its counterpart abroad and the $Y$-industry at home less capital intensive than is $\underline{Y}^{*}$ abroad, as portrayed in (13), the ranking shown in (11) for the general case of such consistency reduces to (14):

$$
\hat{r}_{x}>\hat{p}_{x}>\hat{w}^{*}>\hat{w}>0\left(=\hat{p}_{y}\right)>\hat{r}_{y}
$$

This is the ranking associated with the specific-factors model with the extra detail provided by the ordering of national wage rate changes, both trapped within the bounds set by commodity-price changes. Ranking (14) shows that $\hat{w}^{*}$ lies closer to $\hat{p}_{x}$ than does $\hat{w}$, reflecting $X^{*}$ 's intra-industry labor-intensity ranking vis-a-vis $X$ in (13), while $\hat{w}$ lies closer to $\hat{p}_{y}$ (equal to zero) reflecting $Y^{\prime}$ 's intra-industry labor-intensity vis-a-vis $\underline{Y}^{*}$ in (13).

\footnotetext{
${ }^{8}$ This comparison is equivalent to one among physical capital/labor ratios: $k_{x} / k_{y}>k_{x}^{*} / k_{y}^{*}$. But with reference to the preceding footnote, the ranking $k_{x}>k_{x}^{*}$ need not imply that $\theta_{K X} / \theta_{L X}$ exceeds $\theta_{K X}^{*} / \theta_{L X}^{*}$ since $w$ differ between countries. Similarly, a comparison of $k_{x}$ with $k_{y}$ within a single country need not correspond to the share comparison since each sector uses a different kind of capital. Recall that in the neighborhood production structure two sectors jointly employ at most a single factor.
} 
Although this ranking has intuitive appeal in connection with the specific-factors model, it is not a necessary outcome, and will be violated if one country's capital share exceeds the other's sector by sector. To pursue the analysis, note that both wage rate changes either lie trapped between the price changes or they both lie outside, since within each disaggregated part of the world economy the changes in both wage rates must lie on opposite sides of the commodity price change than does the change in the common return to that type of internationally-mobile capital. Therefore it is useful to concentrate on the behaviour of wage rates, where national labor forces are the "edge" factors in this case.

The procedure we now follow is familiar from our general treatment. Favored world $X$-production consists of home and foreign production, and the pair of competitive profit conditions in these sectors provides a relationship between "edge" returns $\hat{w}$ and $\hat{w}^{*}: 9$

$$
\left(\theta_{K X} \theta_{L X}^{*}\right) \hat{w}^{*}-\left(\theta_{L X} \theta_{K X}^{*}\right) \hat{w}=\left(\theta_{K X}-\theta_{K X}^{*}\right) \hat{p}_{x} .
$$

Similarly, in the fixed-price $Y$-part of the world economy, ${ }^{10}$

$$
-\left(\theta_{K Y} \theta_{L Y}^{*}\right) \hat{w}^{*}+\left(\theta_{L Y} \theta_{K Y}^{*}\right) \hat{w}=0
$$

Figure 4, showing these relationships between wage changes, is drawn under the assumptions both that the multilateral ranking in (12) holds (whereby $X$ is multilaterally capital intensive and $\underline{X}^{*}$ labor intensive), as well as the consistent pair of bilaterial rankings provided by (13). If there were to be no price change in the $X$-sectors of the world economy, the returns to national wage rates would be restricted to the $X X^{*}$ locus. That is, any increase in home $w$ would drive down the world's return to type- $X$ capital, and thus raise foreign $\underline{w}^{*}$. That this line is flatter than the $45^{\circ}$ line reflects the assumption that in a bilateral intra- $X$-industry comparison $X^{*}$ is labor intensive, requiring $\hat{w}^{*}$ to lie closer to the price change (zero along $X X^{*}$ ) than does $\hat{w}$. Similarly the $Y Y^{*}$ locus, drawn for a constant price of $\underline{Y}$, shows $\underline{w}$ and $\underline{w}^{*}$ moving up or down together. Since we are assuming $\underline{Y}$ to be bilaterally labor-intensive compared to $Y^{*}$, this line must be steeper than the $45^{\circ}$ line so that $\hat{w}$ lies closer to $Y$ 's price change (zero) than does $\hat{w}^{*}$. The relationship of each locus to the $45^{\circ}$ line thus reflects the bilateral rankings in (13), while the fact that $Y Y^{*}$ is drawn steeper than $X X^{*}$ is reflective of the multilateral ranking in (12).

An increase in the world price of $X$ shifts the $X X^{*}$ locus upwards to $\left(X X^{*}\right)^{\prime}$; equation (15) confirms this result if the home country's $X$-sector is bilaterally capital-intensive relative to that abroad, as assumed. (This intensity condition thus serves double duty: it implies that $X X^{*}$ is flatter than the $45^{\circ}$ line and that an increase in $p_{x}$ shifts the locus upwards). The amount of the shift, $\left(\theta_{K X}-\theta_{K X}^{*}\right) \hat{p}$, is smaller than the price rise. The latter,

\footnotetext{
${ }^{9}$ The general competitive profit conditions of change in each favored sector are shown in (6).

${ }^{10}$ This corresponds to equation (7) in the general case.
} 


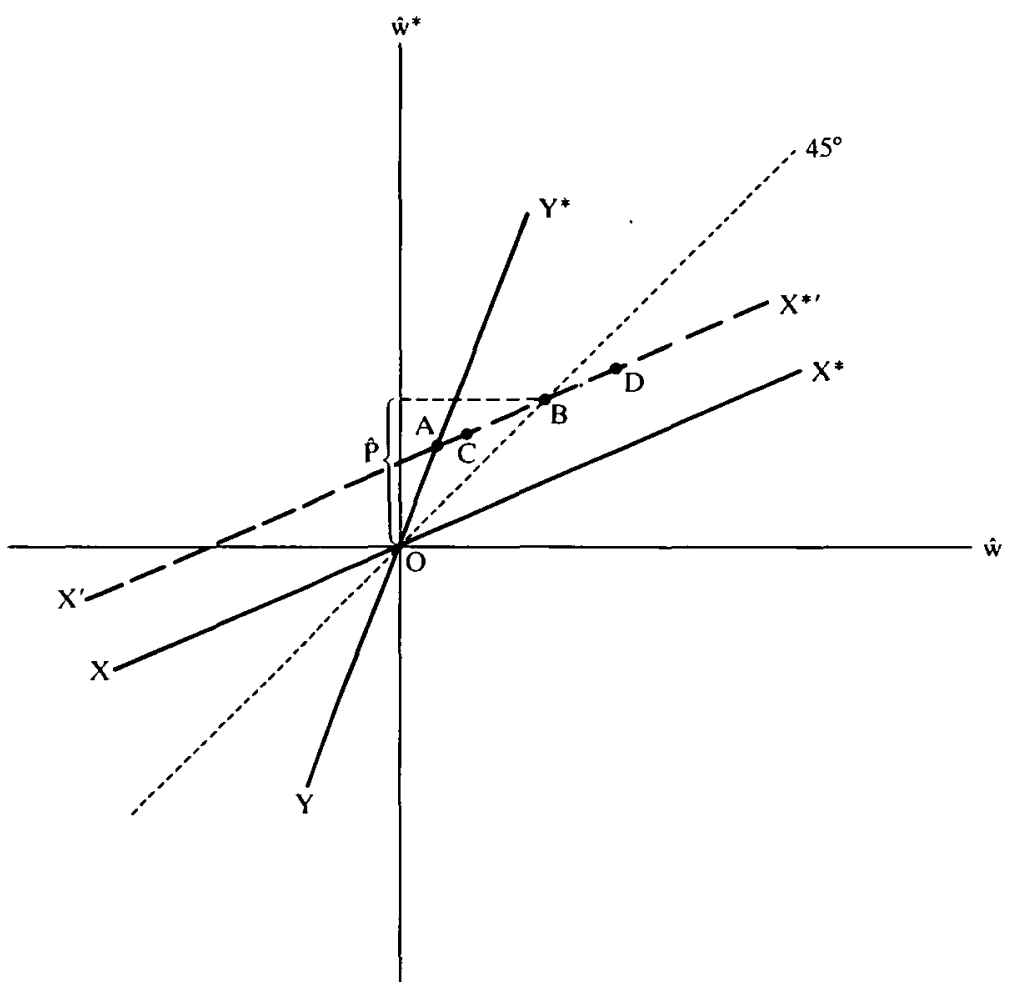

Fig. 4

$\hat{p}$, is shown by the vertical distance to point $\underline{B}$ where $\left(X X^{*}\right)^{\prime}$ intersects the $45^{\circ}$ line; if $\hat{w}$ and $\hat{w}^{*}$ are equal at a point $(B)$ along $\left(X X^{*}\right)^{\prime}$, they must each equal $\hat{p}$. The position of point $A$, where the $\underline{X}^{\prime}$ and $\underline{Y}$ loci intersect, confirms the specific-factors type of result shown in ranking (14) whereby both wage rates rise, but not by as much as the world price of $X$, as well as the intra-industry intensity comparisons whereby $\hat{w}^{*}$ exceeds $\hat{w}$.

Suppose, now, that the multilateral ranking shown by (12) remains valid, but that the difference between the intensities with which the home and foreign $Y$-sectors use labor and capital starts to diminish. The $Y Y^{*}$ locus in Fig. 4 would be shown rotated clockwise (around the origin) from its original position, so that as $p_{x}$ rises the equilibrium changes in wage rates move from $\underline{A}$ to $C$. Factor intensity rankings are still "consistent", supporting again the rankings of returns shown in (14), but both $\hat{w}$ and $\hat{w}^{*}$ move closer to the price change in the $X$-sector, $\hat{p}$. Should home and foreign factor intensity differences in the $Y$-industry completely vanish (leading to a $Y Y^{*}$ schedule co-existent with the $45^{\circ}$ line and an intersection with $\left(X X^{*}\right)^{\prime}$ at $\left.\underline{B}\right)$, both wage rates rise by the same relative amount as does the world price of commodity $X$. Identical value intensities within the world's $Y$-industry require that any wage change in one country be matched 
exactly by a comparable change abroad. With intra-industry factor intensities still assumed to differ in the $X$-part of the world economy, wage rate changes cannot be equal unless they both equal the change in the rate of return to $X$-type capital (and thus to the change in the price of $X$ ).

This type of argument can now easily be extended to show how "inconsistent" intensity rankings must cause national labor forces to become "extreme" in the older Heckscher-Ohlin sense of reflecting an unambiguous improvement (or worsening) of real wages as a consequence of a price change. Suppose the home country's $\underline{Y}$-sector now becomes more capital-intensive than $\underline{Y}^{*}$ abroad, but with the difference less pronounced than in the world's $X$-industry. That is, in Fig. 4 the $Y Y^{*}$ locus is now flatter than the $45^{\circ}$ line (but still steeper than the $X X^{*}$ locus). Intersection is at a point such as $\underline{D}$. The increase in the world price of $\underline{X}$ has raised both countries' wage rates by a more than proportional amount. With each foreign sector bilaterally more labor-intensive than its home counterpart, $\hat{w}^{*}$ must lie closer both to zero and to $\hat{p}$ then does $\hat{w}$, and thus must now fall short of $\hat{w}$. The return to the world's type of capital used exclusively in the favored $X$-part of the world economy cannot rise by as much as the price of $\underline{X}$. Indeed, the return to $X$-type capital may actually fall.

The major results for this interpretation of the neighborhood model for $n=4$ can now be brought together. When the prices of $X$-type goods rise throughout the world, the effect on returns to internationally-mobile but sector-specific capital and occupationally-mobile but country-specific labor depends crucially on the "consistency" of the bilateral intra-industry intensity rankings. The neighborhood production structure requires a multilateral factor-intensity reversal between countries: on a multilateral basis if $\underline{X}$ is capital intensive, $X^{*}$ must be labor intensive (and $\underline{Y}$ must be labor intensive and $\underline{Y}^{*}$ capital intensive). We have defined "consistency" as the situation $n$ in which each intra-industry ranking conforms with the multilateral ranking, implying that each country possesses a sector which is bilaterally capital intensive relative to its counterpart in the other country. In such a case a rise in the world price of type- $X$ products leads to results familiar from specific-factor models: $X$-type capital unambiguously gains and $Y$-type capital loses, while each country's wage rate is trapped between the price changes. However, one country's wage will rise by more than the other, and the winner in this international wage comparison is the country in which the favored $X$-industry is labor intensive.

Lack of "consistency" in the neighborhood model is possible. It is associated with a situation in which one country has an intra-industry dominance in capital's distributive share in both sectors. In such a case relative price changes lead to unambiguous gains or losses to both countries' labor forces, a situation reminiscent of $2 \times 2$ Heckscher-Ohlin models. The key question centers on a comparison of the ratio of capital/labor shares in one industry and the other. If the favored industry has the larger intra-industry intensity spread (so that its bilateral ranking corresponds to 
the multilateral factor intensity ranking), real wages in both countries unambiguously rise. ${ }^{11}$ This rise is especially pronounced in the country which uses labor relatively unintensively in the favored sector. ${ }^{12}$

To stress the point that factor returns in this model are guided by world intra-industry intensity comparisons instead of the more typical intracountry comparisons of intensities between commodities in different industries, note that in the example shown in Fig. 4 in which the $Y Y^{*}$ schedule goes from the origin through point $D$ it is possible for the $X$-sector to exhibit a higher capital intensity than the $Y$-sector in each country. For example, suppose

$$
\frac{\theta_{K X}}{\theta_{L X}}>\frac{\theta_{K Y}}{\theta_{L Y}}>\frac{\theta_{K X}^{*}}{\theta_{L X}^{*}}>\frac{\theta_{K Y}^{*}}{\theta_{L Y}^{*}},
$$

with, nonetheless, criterion (12) satisfied. Then despite the relative laborintensity in each country of the industry $(Y)$ which has suffered a relative decline in price, real wages in both countries unambiguously rise. The two crucial strands in the argument are: (i) the same country has a higher intra-industry capital share in each sector-this leads to unambiguous real wage changes, and (ii) the industry favored by the price rise exhibits a wider spread in techniques between countries than does the other industry-this requires wages more closely to approximate the price that has risen. Nowhere in the argument is appeal made to intra-country comparisons of techniques.

\section{Concluding remarks}

There is no doubt that the two most heavily used simple general equilibrium models of production in international trade theory and other fields are the $2 \times 2$ model associated with the names of Heckscher, Ohlin, and Samuelson and the $3 \times 2$ specific-factors model. The former stresses the asymmetry in the proportions in which factors of production are utilized, while the latter focuses upon a different asymmetry-that between the degree of intersectoral mobility possessed by each factor. It has been a relatively easy task to free the specific-factors model from its dependence upon a small number of commodities; the $(n+1) \times n$ version of the model preserves most of the properties characteristic of the $3 \times 2$ model. ${ }^{13}$ But the $2 \times 2$ model has not been generalized that easily. Some structure must be

${ }^{11}$ As the analysis in Fig. 4 suggests, as the spread in the world $Y$-industry becomes smaller, for given spread in the world $X$-industry, wage rate changes must more closely resemble the change in the price of $X$ (the favored sector).

${ }^{12}$ Indeed the same country will (in this incosistent case) use labor less intensively sector-by-sector. In our example (point $D$ in Fig. 4), the foreign country's firms are labor intensive in both intra-industry comparisons, so that $\hat{w}^{*}$ is tied closer to both $\hat{p}_{x}$ and $\hat{p}_{y}$ than is $\hat{w}$. This implies $\hat{w}>\hat{w}^{*}$ if wages rise generally more than prices.

${ }^{13} \mathrm{See}$, for example, the analysis in Jones (1975). 
imposed on the $n \times n$ model before many useful comparative statics properties can be obtained. ${ }^{14}$

The present paper suggests a route towards a manageable model in higher dimensions. The neighborhood production structure preserved much of the ease of analysis characteristic of the standard $2 \times 2$ model by limiting the occupational alternatives which any factor of production possesses to two-employment in the two neighboring industries when a circular ranking can be devised such as Fig. 1 . As in any $\underline{n} \times \underline{n}$ model the role of factor intensity comparisons is crucial in linking commodity price changes to the ranking of factor returns. Although in the neighborhood production structure no pair of industries shares the same pair of factors (except in the limiting $2 \times 2$ version), a multilateral factor intensity ranking can be devised whereby each factor is intensively used in a different sector, and such a ranking insures that if the price of the commodity produced in that sector rises (alone), so will the return to the factor used intensively in that sector.

Our strategy was to utilize this model to analyze the effect on factor returns of a price rise common to two neighboring productive sectors, while the rest of the economy remained with prices fixed. ${ }^{15}$ Such an exercise leads to a clear categorization of productive factors: the factor used in both favored sectors is not used anywhere else, a set of $n-3$ factors are used only in the fixed-price part of the economy, while a pair of factors (the "edge" factors) are used in both subdivisions of the economy. The distinctions are reminiscent of that made in the specific-factor model between specific factors and the mobile factor. The potential analogy between the two models prompted the question: Can the factor price rankings associated with neighboring price rises in the $n \times n$ neighborhood productive structure approximate those associated with relative price changes in the specific-factors model? The answer was affirmative if factor intensity rankings in each subdivision of the economy proved "consistent" with the multilateral ranking.

The particular application of the neighborhood production structure considered in this paper allows world trade between two large countries, each with a national but intersectorally mobile labor force and making use of a commpn pool of internationally mobile but sector-specific capitals. This model fits the neighborhood production structure for the $4 \times 4$ case, when the world economy is subdivided into two parts, each representing a particular industry located in both countries. Only if bilateral intra-industry factor intensity rankings are "consistent" will factor returns resemble the specific factors model. As was shown, such a "consistent" ranking is

\footnotetext{
${ }^{14}$ Of course some properties are deducible even in general models. For example the lack of joint production imposes some restrictions. See Ethier (1974) or Jones and Scheinkman (1977) for futher discussion. For an alternative form of $n \times n$ model, with sufficient structure to guarantee generalized Stolper-Samuelson results, see Jones and Marjit (1984).

${ }_{15}$ The same techniques could be used for any single subdivision of the economy, regardless of the number of sectors in the favored part.
} 
violated if one country employs more capital-intensive techniques than the other sector-by-sector (in a bilateral value sense).

As these remarks suggest, the neighborhood production structure is rich in the variety of its outcomes. Factor prices are freed from a direct dependence on factor endowments (arguably a useful characteristic in models with international factor mobility), and both specific-factor and Heckscher-Ohlin type of factor price rankings may be obtained. The choice between the two rests upon a simple test of consistency of bilateral factor intensity rankings with the overall multilateral ranking. Further applications of the model with room for more than four productive activities could be devised since the neighborhood production structure handles higher dimensions with ease.

University of Rochester, N.Y., USA.

Graduate Institute of International Studies, Geneva.

\section{REFERENCES}

BRECHER, R. and Findlay, R. (1983). "Tariffs, Foreign Capital and National Welfare with Sector-Specific Factors," Journal of International Economics, 14, 277-288.

CAves, R. (1971). "International Corporations: the Industrial Economics of Foreign Investment," Economica, 38, 1-27.

CORNES, R. and KIERZKOWSKI, H. (1981). "Inter- and Intra-Industry Factor Specificity in an Open Economy," mimeo.

ETHIER, W. (1974). "Some of the Theorems of International Trade with Many Goods and Factors," Journal of International Economics, 4, 199-206.

JONES, R. W. (1961). "Comparative Advantage and the Theory of Tariffs: A Multi-Country, Multi-Commodity Model," Review of Economic Studies, 28, 161-75.

(1975. "Income Distribution and Effective Portection in a Multi-Commodity Trade Model," Journal of Economic Theory, 11, 1-15.

and DeI, F. (1983). "International Trade and Foreign Investment: a Simple Model," Economic Inquiry, 21, 449-464.

— and MARIT, S. (1984). "A Simple Production Model with Stolper-Samuelson Properties," mimeo.

—, Neary, J. P. and Ruane, F. (1983). "Two-Way Capital Flows: Cross-hauling in a Model of Foreign Investment," Journal of International Economics, 14, 357-66.

— and Scheinkman, J. (1977). "The Relevance of the Two-Sector Production Model in Trade Theory," Journal of Political Economy, 85, 909-935. 